\title{
Analytical Methodologies for the Stereoselective Determination of Sibutramine: An Overview
}

\author{
Alexandru Robert Vlad11, Gabriel Hancu1, Hajnal Kelemen¹, Diana Ciurcă2 ${ }^{1}$, Amelia Tero-Vescan³ \\ 1 Department of Pharmaceutical Chemistry, Faculty of Pharmacy, University of Medicine and Pharmacy, Tîrgu Mureș, Romania \\ 2 Department of Organic Chemistry, Faculty of Pharmacy, University of Medicine and Pharmacy, Tîrgu Mureș, Romania \\ 3 Department of Biochemistry, Faculty of Pharmacy, University of Medicine and Pharmacy, Tîrgu Mureș, Romania
}

Sibutramine is a chiral anti-obesity drug which decreases food intake and increases energy expenditure. In therapy it is used as a racemic mixture; however both pharmacokinetic and pharmacodynamic data have revealed enantioselective behavior of sibutramine and its major active metabolites. Several chromatographic and electrophoretic analytical methods have been published so far for the chiral determination of sibutramine from pharmaceutical preparations and biological samples. The current paper aims to provide a systematic review of the stereochemical aspects and analytical methods used for the enantiodetermination of sibutramine and its active enantiomers covering the last 15 years.

Keywords: sibutramine; chirality; enantioseparation; high perfomance liquid chromatography; capillary electrophoresis

Received 7 May 2017 / Accepted 13 June 2017

\section{Introduction}

Sibutramine $[( \pm)-1-(\mathrm{p}$-chlorophenyl)a-isobutyl-N,N dimethyl cyclobutane methylamine hydrochloride] (SIB) is an oral anorexiant which inhibits central reuptake of serotonin, norepinephrine, and to a lesser extent dopamine; prescribed in the treatment of obesity along with a reduced calorie diet. Unlike other anorexiant agents, like amphetamine, SIB does not affect the release of neurotransmitters [1].

SIB pharmacological mechanism of action by which it exerts its weight-loss effect is due to a combination of feelings of satiety, reduced appetite and induction of thermogenesis [2]. SIB is prescribed as an adjunct in the management of exogenous obesity along with exercise and diet [3].

SIB has a chiral carbon in its molecule and consequently exists in the form of two enantiomers, $R$-SIB and $S$-SIB (figure 1). The substance is marketed as a racemic mixture, but pharmacodynamic and pharmacokinetic profile has revealed the enantioselective behavior of SIB, as $R$-SIB presents a better anorexic effect than racemic SIB or its $S$ enantiomer [4].<smiles>CC(C)C[C@H](N(C)C)C1(c2ccc(Cl)cc2)CCC1</smiles><smiles>CC(C)CC(N(C)C)C1(c2ccc(Cl)cc2)CCC1</smiles>

Fig. 1. Chemical structure of SIB enantiomers

SIB undergoes first-pass demethylation to active metabolites, mono-desmethylsibutramine (MDS) and di-des-

* Correspondence to: Gabriel Hancu

E-mail: gabriel.hancu@umftgm.ro methylsibutramine (DDS) which are primarily responsible for its pharmacologic effects (figure 2). The active metabolites are biotransformed further in the liver and excreted primarily in the urine [5]. SIB metabolites are also chiral, and exhibit enantioselecive effects; as $R$-SIB metabolites exhibit higher potency than the S-SIB metabolites; also $R$ SIB metabolites were present at lower concentrations in the body because of their fast metabolization to hydroxylated and carbamoylglucuronized forms and their fast urinary excretion [6]. Additionaly, another pharmacokinetic study demonstrates that $R$-SIB is biotransformed preferentially over $S$-SIB in primary cultures of rat hepatocytes and in rat liver microsomes [7].

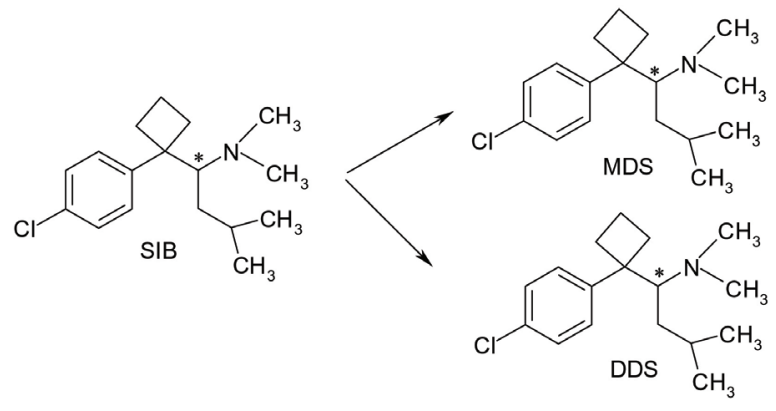

Fig. 2. SIB metabolism (* denotes the chiral centers)

Initially, at doses of $10-20 \mathrm{mg}$ per day, SIB was considered to present good safety profile, as it does not induce cardiovascular adverse effects or pulmonary hypertension, in comparison with other previously used antiobesity agents [8]. However, the results of SCOUT (Sibutramine Cardiovascular and Diabetes Outcome Study) clinical trial, a study designed to evaluate the efficacy/safety ratio of SIB in high-risk patients, showed that long-term treatment with SIB exposed patients with pre-existing cardiovascular disease to a statistically significant increased risk for stroke and myocardial infarction [9]. The European Medicines 
Agency (EMA) recommended in 2010 the suspension of marketing authorizations for SIB across EU countries, taking in consideration reports considering that the cardiovascular risks of SIB seems to outweigh the benefits as a weight-loss agent. In the same year Abbot Laboratories announced the withdrawing SIB from the US market under pressure from the Food and Drug Administration (FDA), citing concerns over therapeutic efficiency coupled with increased risk of adverse cardiovascular adverse effects [10].

Nowadays SIB is still used in more than 40 countries from Asia and Central and South America. Also, the illicit addition of SIB in counterfeit slimming products has been reported, which represents a serious public health problem [11].

The enantioselective behavior of SIB enantiomers and its two major active metabolites have been of great interest from pharmacodynamic and pharmacokinetic point of view. For the development of SIB as a chiral drug and for pharmacokinetic and pharmacologic studies, the enantioselective separation and determination of SIB become of major importance.

A variety of analytical methods (chromatographic, electrophoretic) have been reported in the past decade for the determination of SIB in pharmaceutical preparations and in biological materials. However the large majority of the published methods report achiral analysis of SIB by reversed-phase liquid chromatography (RP-LC) in pharmaceutical products [12], ultra-high-pressure liquid chromatography with diode-array detection in adulterated weight-loss formulations [13], column-switching high performance liquid chromatography (HPLC) in rat serum [14] or liquid chromatography-mass spectrometry (LC-MS/MS) in human plasma [15]. Despite the pharmaceutical importance of the stereoselective analysis of this compound, only a few studies have been reported for chiral separation of SIB and its metabolites.

In the current review, we have summed up the previously published methods in the literature for enantiodetermination of SIB and its active metabolites.

\section{Determination of absolute configuration}

Single crystal X-ray crystallography is considered to be the most powerful structural method for the determination of $3 \mathrm{D}$ structures of the molecules; however while the results of a routine diffraction experiment provides unambiguous determination of the relative configuration of the stereogenic centers in the molecule, determination of absolute configuration can be a more challenging task.

In the literature we can find a few reports for the preparation of SIB or its metabolites enantiomers using: resolution of the racemate with a chiral acid; asymmetric synthesis and chiral column HPLC separation.

Racemic SIB was resolved with dibenzoyl-D-tartaric acid, and the absolute stereochemistry of SIB was determined by single crystal X-ray crystallography of the dibenzoyl D-tartrate. The major active metabolite, MDS, was obtained by demethylation of SIB with diethyl azodicarboxylate, a complete retention of the configuration was observed. Based on single crystal X-ray structural analysis, the $(+)$-isomer of SIB salt has the R configuration, the same as the (+)-MDS salt obtained by demethylation. Enantiomeric purity of SIB was verified by HPLC using a chiral stationary phase column (ES-OVM) [16].

Because of the potentially explosive diethyl azodicarboxylate, application of the SIB demethylation method to the mass production of MDS cannot be viable a solution. Consequently another synthesis method of the enantiomerically pure $R$-MDS and $S$-MDS was developed along with an improved alternative synthesis of the racemic MDS. This route was used for kilo-scale production of enantiomerically pure $R$ - and $S$-MDS. Racemic MDS was resolved with either $R$ - or $S$-mandelic acid, and the absolute stereochemistry of MDS was determined by single $\mathrm{X}$-ray crystallography of its mandelate salt. Based on single-crystal X-ray structural analysis, the same results were obtained as in the previous study, as the (+)-isomer of the MDS salt presented $R$-configuration [17].

In order to find an efficient high yielding synthesis method for $R$-MDS, efforts were focused towards the development of a catalytic asymmetric method. The key step in the asymmetric synthesis of $R$-MDS was the enantioselective catalytic addition of iBuLi to aldimine 3 derived from methyl amine and 1-(4-chlorophenyl) cyclobutanecarboxaldehyde [18].

The synthesis of the other enantiopure metabolite DDS was also resolved by combining isolation, purification, and salt formation resolution in one-step, in which chemically pure racemic DDS was isolated from the reaction mixture as a tartaric acid salt, to perform simple crystallizations, in order to provide both enantiopure $R$ - and S-DDS [19].

\section{Chiral separation of sibutramine by chromatographic methods}

Two HPLC techniques can be used for the chiral separation of pharmaceutical substances: indirect and direct ones. The indirect HPLC technique involves the use of a chiral derivatization reagent with the formation of two diastereomer, which can be separated using achiral chromatographic conditions. On the other hand, the direct HPLC technique utilizes a chiral selector added either in the chiral stationary phase or in the mobile phase, and are the most frequently used methods because of their simplicity and rapidity [20].

Several reports have been published for the chiral determination of SIB by HPLC techniques, using protein based chiral stationary phase [21], Chiracel OD column [22] or Chiralcel AGP-stationary phase column [23,24].

The enantioseparation of SIB was achieved by direct HPLC on a Chiralcel OD column $(250 \times 4.6$ $\mathrm{mm}, \quad 10 \mu \mathrm{m})$ using a mobile phase consisting of hexane:ethanol:trifloroacetic acid (93:7:0.05, v/v/v) and a flow rate of $1 \mathrm{~mL} / \mathrm{min}$. The chiral separation of the two 
enantiomers was obtained within 15 minutes with UV detection at a wavelength of $225 \mathrm{~nm}$ [22].

A rapid and simple chromatographic method for the separation of $R$ - and $S$-isomers of SIB, MDS, and DDS in rat plasma by LC-MS/MS using a Chiral-AGP stationaryphase column (chiral stationary-phase based on bonding to $\alpha$-acid glycoprotein -AGP) $(100 \times 2.0 \mathrm{~mm}, 5 \mu \mathrm{m})$ has been also reported. The enantiomers of SIB and its two major active metabolites were extracted from rat plasma using diethyl ether and $\mathrm{n}$-hexane under alkaline conditions. After evaporation the organic layer, the residue was reconstituted in the mobile phase $(10 \mathrm{mM}$ ammonium acetate buffer adjusted to $\mathrm{pH} 4.03$ with acetic acid:acetonitrile, $94: 6, \mathrm{v} / \mathrm{v})$. The method was used to characterize the plasma concentrations of the stereoisomers of SIB and its metabolites in rat plasma, following the oral administration of a single oral dose of $10 \mathrm{mg} / \mathrm{kg}$ of racemic SIB [23].

The same optimized LC-MS/MS method was then applied for the determination of SIB and its two active metabolites in human plasma. The method was validated in accordance with FDA regulations for the validation of bioanalytical methods. The method was successfully applied to quantify SIB and its metabolites stereoisomers plasma concentration in healthy volunteers [24].

\section{Chiral separation of sibutramine by electrophoretic methods}

The use of chiral capillary electrophoresis (CE) in enantiomeric separations has gained momentum in the chiral analysis of pharmaceutical substances, with advantages related to the rapid method development and high separation efficiency; CE is also a highly flexible and versatile analytical method, with a minimal use of chiral selectors and analytes, and usually uses a direct method of separation by simply adding the chiral selector to the background electrolyte [25].

Cyclodextrins (CDs) are the most frequently used chiral selectors in enantiomeric separations by $\mathrm{CE}$, because several useful properties in chiral CE such as good complexation ability of various analytes, acceptable solubility in water, low UV absorption, commercial availability, relatively low price and good chemical stability [26].

Capillary zone electrophoresis (CZE) using derivatized neutral CD - methyl- $\beta$-CD (M- $\beta$-CD) or derivatized ionized CD - carboxymethyl- $\beta-C D$ as chiral (CM- $\beta-C D)$ selectors was the first-choice method used in several studies for the enantioseparation of SIB enantiomers [27-30].

Separation of SIB enantiomers by CE was accomplished using a mixed buffer composition containing a mixture of $20 \mathrm{mM}$ phosphate and $10 \mathrm{mM}$ citrate and either $5 \mathrm{mM}$ $\mathrm{M}-\beta-\mathrm{CD}(\mathrm{pH} 4.3)$ or $5 \mathrm{mM}$ CM- $\beta-\mathrm{CD}(\mathrm{pH} 6.5)$ as chiral selectors. The separation was achieved on a $50 \mathrm{~cm} \times 50 \mu \mathrm{m}$ fused silica capillary, UV detection was used at $223 \mathrm{~nm}$. During the method optimization, the mixed buffer showed superior separation results in comparison with single component buffers. The stability constants of $R$ - and S-SIB demonstrated that the resolution of SIB enantiomers was primarily due to the difference in the stability constants of the complexes. The optimized CE method was used for the determination of SIB enantiomers in pharmaceutical formulations [27].

Proton nuclear magnetic resonance spectroscopy $\left({ }^{1} \mathrm{H}-\right.$ NMR) and CE have been used to discriminate SIB enantiomers using different $\mathrm{CD}$ derivatives as chiral selectors. Possible correlations between ${ }^{1} \mathrm{H}-\mathrm{NMR}$ and $\mathrm{CE}$ results were examined; good correlation between the H-NMR shift non-equivalence data for SIB and the enantioseparation by CE was observed. Separation of SIB enantiomers by $\mathrm{CE}$ was achieved using a $50 \mathrm{mM}$ of phosphate buffer at $\mathrm{pH} 3.0$ and $10 \mathrm{mM}$ of $\mathrm{M}-\beta-\mathrm{CD}$ as chiral selector. The $\mathrm{CE}$ method was optimized by a complex study regarding the influence of buffer concentration, buffer $\mathrm{pH}, \mathrm{CD}$ concentration, and system temperature on the chiral resolution of SIB enantiomers. The analytical performance of the method was verified; the method was validated according to $\mathrm{ICH}$ guidelines and was applied for the quantitative determination of SIB enantiomers in pharmaceutical formulations. On a $600 \mathrm{MHz}{ }^{1} \mathrm{H}-\mathrm{NMR}$ analysis, enantiomer signal separation of SIB was obtained by fast diastereomeric interaction with the chiral selector. Using ${ }^{1} \mathrm{H}-\mathrm{NMR}$ and 2D ROESY studies a structure of the inclusion complex was also proposed [28].

In another study a complex screening of a large number of native and derivatized, neutral and ionized CD derivatives was performed in order to establish the optimum chiral selector for the enantiodetermination of SIB. During the optimization process, the effects of buffer composition, background electrolyte concentration and $\mathrm{pH}, \mathrm{CD}$ type and concentration, applied voltage, capillary temperature and injection parameters on the chiral resolution were studied. The best results on a short fused silica capillary of $30 \mathrm{~cm} \times 50 \mu \mathrm{m}$ were obtained using a $50 \mathrm{mM}$ phosphate buffer containing $10 \mathrm{mM}$ randomly methylated $\beta$-CD (RAMEB) as chiral selector at a $\mathrm{pH}$ of 4.50 with UV detection at $220 \mathrm{~nm}$; the separation of enantiomers was achieved in approximately 5 minutes [29].

SIB was used as the model substance in order to demonstrate the reversal of enantiomer migration order in $\mathrm{CE}$ separations in correlation with CD concentration. In the presence of $\mathrm{M}-\beta-\mathrm{CD}$ and 2-hydroxypropyl- $\beta-\mathrm{CD}$ (HP$\beta$-CD) overlapping of migration for SIB enantiomers was observed, while reversal of enantiomer migration order was observed in the case of $\beta-C D$ and acetyl- $\beta-C D$ (A- $\beta-C D)$ when increasing $C D$ concentration. The reversal of enantiomer migration order could be explained in terms of the opposing effects of the stability and the limiting complex mobility of the SIB-CD complexes while the enantioseparation of SIB with $\mathrm{M}$ - and HP- $\beta-C D$ was based on the differences in the binding constants of the SIB-CD complexes while [30].

SIB was one of the ten model compounds used for the testing of an open-tubular capillary electrochromatogra- 
phy column prepared by chemically immobilized $\beta-C D$ modified gold nanoparticles onto a new surface with prederivatization of (3-mercaptopropyl)-trimethoxysilane. To improve enantioselectivity, $\beta-\mathrm{CD}$ was used as chiral additive in order to identify synergistic effects and increase chiral resolution [31].

\section{Conclusions}

In therapy, SIB has been used as a racemic mixture, while the pure enantiomers of the compound have not been available. For efficiency and safety reasons, maybe SIB may have been developed and administered as a pure enatiomer, and for the evaluation of pharmacokinetic and pharmacodynamic profile enantioselective determination of SIB enantiomers is needed. Without doubt HPLC methods have proven to be the most reliable and versatile analytical techniques for chiral measurements of pharmaceutical substances, however CE has become a viable alternative and a complementary technique for certain applications because of its high separation efficiency, relatively short analysis time, and low consumption of samples and reagents.

\section{References}

1. Luque CA, Rey JA - The discovery and status of sibutramine as an antiobesity drug. Eur J Pharmacol. 2002;(2-3):119-128.

2. Finer N. - Sibutramine: its mode of action and efficacy. Int $J$ Obes Relat Metab Disord. 2002;26(4):29-33.

3. Nisoli E, Carruba MO - An assessment of the safety and efficacy of sibutramine, an anti-obesity drug with a novel mechanism of action. Obes Rev. 2000;1(2):127-139.

4. Bodhankar SL, Thakurdesai PA, Singhal S, et al. - Anorexic effect of (R)-sibutramine: comparison with (RS)-sibutramine and (S)-sibutramine. Indian J Physiol Pharmacol. 2007;51(2):175-178.

5. Filippatos TD, Kiortsis DN, Liberopoulos EN, et al. - A review of the metabolic effects of sibutramine. Curr Med Res Opin. 2005;21(3):457468.

6. Glick SD, Haskew RE, Maisonneuve IM, et al. - Enantioselective behavioral effects of sibutramine metabolites. Eur J Pharmacol. 2000;397:93-102.

7. Noh K, Bae K, Min B, et al. - Enantioselective pharmacokinetics of sibutramine in rat. Arch Pharm Res. 2010;33:267-273.

8. Tyczynski JE, Oleske DM, Klingman D, et al. - Safety assessment of an anti-obesity drug (sibutramine): a retrospective cohort study. Drug Saf. 2012;35(8):629-644.

9. Maggioni AP, Caterson ID, Urso R, et al. - Relation between weight loss and causes of death in patients with cardiovascular disease: finding from the SCOUT trial. J Cardiovasc Med. 2017;18(3):144-151.

10. Scheen AJ - Cardiovascular risk-benefit profile of sibutramine. Am J Cardiovasc Drugs. 2010; 10(5):321-334.

11. Dunn JD, Gryniewicz-Ruzicka CM, Kauffman JF, et al.- Using a portable ion mobility spectrometer to screen dietary supplements for sibutramine. J Pharm Biomed Anal. 2011; 54(3):469-474.

12. Diefenbach IC, Friedrich M, Dos Santos MR, et al. - Development and validation of a column high-performance liquid chromatographic method for determination of sibutramine in capsules. J AOAC Int. 2009; 92(1):148-151.
13. Rebiere H, Guinot P, Civade C, et al. - Detection of hazardous weightloss substances in adulterated slimming formulations using ultra-highpressure liquid chromatography with diode-array detection. Food Addit Contam Part A Chem Anal Control Expo Risk Assess. 2012;29(2):161171.

14. Um SY, Kim KB, Kim SH, et al. - Determination of the active metabolites of sibutramine in rat serum using column-switching HPLC. J Sep Sci. 2008;31(15):2820-2826.

15. Bae JW, Choi Cl, Jang CG, et al. - Simultaneous determination of sibutramine and its active metabolites in human plasma by LC-MS/MS and its application to a pharmacokinetic study. Biomed Chromatogr. 2011;25(11):1181-1188.

16. Fang QK, Senanayake $\mathrm{CH}$, Han Z, et al. - First preparation of enantiomerically pure sibutramine and its major metabolite, and determination of their absolute configuration by single crystal X-ray analysis. Tetrahedron Asymmetry, 1999; 10:4477-4480.

17. Han Z, Krishnamurthy D, Pflum D, et al. - First practical synthesis of enantiomerically pure (R)- and (S)-desmethylsibutramine (DMS) and unambiguous determination of their absolute configuration by singlecrystal X-ray analysis. Tetrahedron Asymmetry, 2002;13(2):107-109.

18. Krishnamurthy D, Han Z, Wald SA, et al. - First asymmetric synthesis of (R)-desmethylsibutramine. Tetrahedron Asymmetry, 2002;43(13):23312333.

19. Han Z, Krishnamurthy D, Fang QF, et al. - New resolution approach for large-scale preparation of enantiopure didesmethylsibutramine (DDMS). Tetrahedron Asymmetry, 2003;14(22):3533-3536.

20. Bojarski J, Aboun-Enein HY, Ghanem A - What's new in chromatographic enantioseparations. Curr Anal Chem. 2005;1:59-97.

21. Singh AK, Pedro LG, Gomes FP, et al. - Development and validation of sensitive methods for determination of sibutramine hydrochloride monohydrate and direct enantiomeric separation on a protein-based chiral stationary phase. J. AOAC Int. 2008; 91(3):572-579.

22. Radhakrishna T, Lakshmi-Narayana C, Sreenivas-Rao D, et al. - LC method for the determination of assay and purity of sibutramine hydrochloride and its enantiomers by chiral chromatography. J Pharm Biomed Anal. 2000;22:627-639.

23. Bae K, Noh K, Jang K, et al. - Analysis of enantiomers of sibutramine and its metabolites in rat plasma by liquid chromatography-mass spectrometry using a chiral stationary-phase column. J Pharm Biomed Anal. 2009;50(2):267-70.

24. Kang W, Bae K, Noh K - Enantioselective determination of sibutramine and its active metabolites in human plasma. J Pharm Biomed Anal. 2009; 51:264-267.

25. Scriba GK - Differentiation of enantiomers by capillary electrophoresis. Top Curr Chem. 2013; 340:209-275.

26. Rezanka P, Navrátilová K, Rezanka M, et al. - Application of cyclodextrins in chiral capillary electrophoresis. Electrophoresis. 2014; 35(19):27012721.

27. Zhu H, Wu E, Chen J, et al. - Enantioseparation and determination of sibutramine in pharmaceutical formulations by capillary electrophoresis. Bull. Korean Chem. Soc. 2010; 31:1496-1500.

28. Lee YJ, Choi S, Lee J, et al. - Chiral discrimination of sibutramine enantiomers by capillary electrophoresis and proton nuclear magnetic resonance spectroscopy. Arch Pharm Sci Res. 2012;35(4):671-681.

29. Hancu G, Hilochie A, Vlad RA, et al. - Enantiomeric separation of sibutramine by capillary zone electrophoresis. J Braz Chem Soc. 2016;27(6):1116-1120.

30. Zhu H, Wu E, Chen J, et al. - Reverse migration order of sibutramine enantiomers as a function of cyclodextrin concentration in capillary electrophoresis. J Pharm Biomed Anal. 2011; 54:1007-1112.

31. Fang L, Yu J, Jiang Z, et al. - Preparation of a $\beta$-cyclodextrin-based open-tubular capillary electrochromatography column and application for enantioseparations of ten basic drugs. PLoS One. 2016; 15(1): e0146292. 